STRUCTURAL BIOLOGY

ISSN 2059-7983

Received 6 January 2018

Accepted 19 March 2018

Edited by S. Wakatsuki, Stanford

University, USA

Keywords: automatic data processing; microcrystals; KAMO; small-wedge data sets.

Supporting information: this article has supporting information at journals.iucr.org/d

\section{KAMO: towards automated data processing for microcrystals}

\author{
Keitaro Yamashita, Kunio Hirata and Masaki Yamamoto*
}

RIKEN SPring-8 Center, Sayo 679-5148, Japan. *Correspondence e-mail: yamamoto@riken.jp

In protein microcrystallography, radiation damage often hampers complete and high-resolution data collection from a single crystal, even under cryogenic conditions. One promising solution is to collect small wedges of data $\left(5-10^{\circ}\right)$ separately from multiple crystals. The data from these crystals can then be merged into a complete reflection-intensity set. However, data processing of multiple small-wedge data sets is challenging. Here, a new open-source dataprocessing pipeline, $K A M O$, which utilizes existing programs, including the $X D S$ and $C C P 4$ packages, has been developed to automate whole dataprocessing tasks in the case of multiple small-wedge data sets. Firstly, KAMO processes individual data sets and collates those indexed with equivalent unitcell parameters. The space group is then chosen and any indexing ambiguity is resolved. Finally, clustering is performed, followed by merging with outlier rejections, and a report is subsequently created. Using synthetic and several realworld data sets collected from hundreds of crystals, it was demonstrated that merged structure-factor amplitudes can be obtained in a largely automated manner using $K A M O$, which greatly facilitated the structure analyses of challenging targets that only produced microcrystals.

\section{Introduction}

The major difficulty in solving protein structures by X-ray crystallography is obtaining crystals with suitable size and sufficient diffracting power. Recently, X-ray microbeams have become available at synchrotron light sources and have enabled data collection from microcrystals at high signal-tonoise ratios (Smith et al., 2012; Owen et al., 2016; Yamamoto et al., 2017). The preparation of microcrystals is often easier than that of larger crystals, and smaller crystals may sometimes be better ordered and thus lead to higher quality data sets (Cusack et al., 1998; Evans et al., 2011). The lipidic mesophase method has facilitated the production of small but high-quality crystals of membrane proteins, which are typically difficult targets in protein crystallography (Caffrey, 2015). However, the radiation-dose limit of greater than $10 \mathrm{MGy}$ is rapidly exceeded in microcrystals, and consequently multiple crystals are needed to overcome this problem. Serial femtosecond crystallography (SFX) using an X-ray free-electron laser (XFEL; Schlichting, 2015) is a very powerful approach in microcrystallography, based on the principle of 'diffraction before destruction'. Since an ultra-intense femtosecond XFEL pulse only permits the collection of a single still diffraction image, thousands to tens of thousands of diffraction patterns are usually required for structural analysis. Although data collection from such a large number of crystals is fast at a highrepetition-rate XFEL, limited beamtime prevents its routine use. In contrast, radiation damage from synchrotron radiation cannot be completely avoided but high-resolution diffraction 
images can be collected via the rotation method, where integrated Bragg intensities are experimentally obtained. At cryogenic temperatures, small-wedge $\left(5-10^{\circ}\right)$ data collection can be a good compromise to obtain strong diffraction signals under tolerable doses, and tens to hundreds of data sets are usually sufficient to obtain a high-resolution structure. Efficient data collection can be achieved and easily automated when multiple microcrystals are held in a sample holder. MeshAndCollect, an automated multi-crystal data-collection workflow, has been developed at the ESRF (Zander et al., 2015). The recently developed $Z O O$ system (Hirata et al., manuscript in preparation) at the microfocus beamline BL32XU at SPring-8 also supports the automatic collection of small-wedge data sets from loop-harvested microcrystals. These systems enabled rapid data collection from a large number of crystals. Multi-crystal data-collection strategies are advantageous over single-crystal data collection in obtaining accurate data which allow SAD phasing when correctly processed (Akey et al., 2014; Liu \& Hendrickson, 2015; Huang et al., 2016; Olieric et al., 2016). Small-wedge data collection is also compatible with room-temperature experiments that utilize crystallization plates, without the need for tedious crystal harvesting and cooling, as well as possibly allowing the exploration of functionally relevant protein conformations (Fraser et al., 2011).

In small-wedge data collection, data processing is more complicated than processing single-crystal data. Although the indexing and integration of individual wedges may be straightforward, there are some potential issues to take note of, some of which are now listed: (i) space-group determination can be challenging for novel structure determination and any existing indexing ambiguity (Brehm \& Diederichs, 2014) must be resolved, (ii) clustering may also be required to identify non-isomorphous data sets prior to merging and (iii) during merging, rogue data sets that deteriorate the overall data quality should be detected and removed. To help to address the aforementioned issues, several key technologies have been developed, including automated data-processing pipelines that process individual wedges, such as xia2 (Winter, 2010) and autoPROC (Vonrhein et al., 2011). The optimized use of the $X D S$ package for processing in situ diffraction data sets has been described previously (Huang et al., 2015) and methods to resolve the indexing ambiguity have been developed for SFX (Brehm \& Diederichs, 2014; Kabsch, 2014). Regarding clustering, two major methods have been presented, one based on the similarity of unit-cell parameters (Foadi et al., 2013) and the other on the correlation coefficients of intensities (Giordano et al., 2012). Yet another correlation-based approach that performs a multidimensional scaling analysis to sort out data by systematic differences has also been suggested (Diederichs, 2017). As the selection of isomorphous data is a combinatorial problem, the use of a genetic algorithm has been proposed (Zander et al., 2016). To identify rogue data sets, a method based on $\mathrm{CC}_{1 / 2}$ has been presented (Assmann et al., 2016). Despite the welcome advances in technology, the workflow as a whole entity has yet to be automated to the best of our knowledge, which is important for high-throughput analysis and should vastly aid decision making during beamline experiments.

Presented here is a new data-processing pipeline, KAMO (which in Japanese means a mallard, Anas platyrhynchos), which has been developed to automate the whole dataprocessing procedure for multiple small-wedge data sets by using existing programs including XDS (Kabsch, 2010), DIALS (Winter et al., 2018) and CCP4 (Winn et al., 2011). $K A M O$ was originally developed for online use at the SPring- 8 beamlines, but is now publicly available under an open-source license and can be used anywhere for small-wedge data sets. $K A M O$ has already been used in several structure determinations from multiple microcrystals (Abe et al., 2017; Shihoya et al., 2017; Taniguchi et al., 2017; Lee et al., 2017; Miyauchi et al., 2017; Suno et al., 2017). In this article, the details of KAMO are described, followed by processing examples using synthetic and real-world data sets for the mercury-bound $\mathrm{M}_{2}$ receptor and cypovirus polyhedra, whereby the merged structure-factor amplitudes can be obtained with minimal user intervention using $K A M O$.

\section{KAMO functions for multiple small-wedge data}

\subsection{Program overview}

$K A M O$ has been developed to automate the data processing of multiple wedges and merging. For this purpose, the processing of individual data sets, the selection of data sets belonging to the same lattice, clustering, merging with outlier rejections and the creation of a report were implemented. The workflow of $K A M O$ is shown in Fig. 1. KAMO functionality was implemented in the yamtbx package (https:/github.com/ keitaroyam/yamtbx), which depends on the $c c t b x$ library (Grosse-Kunstleve et al., 2002). The header information of several diffraction-image formats is obtained using a modified version of the $X I O$ module originally included in the $x d s m e$ package (P. Legrand; https://github.com/legrandp/xdsme). Other dependent Python libraries include networkx for graph algorithms (Hagberg et al., 2008) and SciPy for optimization and clustering (Jones et al., 2001). The XDS package (Kabsch, $2010)$ is used for data processing: $X D S$ for the indexing and integration of wedges and XSCALE for scaling and merging. From the CCP4 program suite (Winn et al., 2011), POINTLESS (Evans, 2011), CTRUNCATE and BLEND (Foadi et al., 2013) are used. DIALS (Winter et al., 2018) is optionally supported for indexing and integration.

$K A M O$ was originally developed for use at the SPring-8 beamlines, especially with the $Z O O$ system. In the online mode, $K A M O$ initiates data processing once all image files have become available by monitoring the $\log$ files of $B S S$, the standard data-collection program at SPring-8 (Ueno et al., 2005). For offline use, the $b l=0$ ther option can be specified to find data sets from the filesystem.

\subsection{Data processing of individual wedges}

The processing of individual wedges is controlled through a GUI. When the kamo command is started, the files in the 
subdirectories are identified and by default the processing results are saved in the _kamoproc/ directory. The jobs are run in parallel using a queuing system (the Sun Grid Engine is currently supported) or on a local computer.

Indexing, integration and non-empirical intensity corrections are performed using $X D S$ by default. In the preparation of XDS. INP, instrumental geometries, including the incident beam, goniometer and detector, are recognized from the image file header in the same way as for generate XDS.INP (https://strucbio.biologie.uni-konstanz.de/xdswiki/ index.php/Generate_XDS.INP). There is an option to use $d x t b x$ (Parkhurst et al., 2014) as implemented in $c c t b x$, which may be more generic. KAMO does not usually assume that the unit-cell parameters and space-group symmetry are known $a$ priori. Indexing is performed without any prior knowledge and integration is performed with $P 1$ symmetry.

Known unit-cell parameters can optionally be used. In this case, they are used in indexing if an attempt without this information has failed. The integration will not be performed if the indexing result is inconsistent with the known unit cell. Providing known unit-cell parameters that are slightly incorrect can sometimes lead to an improper indexing result and users should be careful when using this option.

Scaling (JOB=CORRECT) is performed based on the symmetry deduced by POINTLESS (Evans, 2011). Nonempirical corrections, that is polarization and absorption by air and the sensor, are only applied in the CORRECT job with the CORRECTIONS= option, and the corrected intensity set is saved as XDS_ASCII.HKL_noscale. This file is used in merging where empirical corrections are performed. POINTLESS is used again for the XDS_ASCII.HKL file, and if the estimated Laue symmetry differs from that estimated from INTEGRATE. HKL, the candidate with higher probability is accepted. However, symmetry estimation may be less reliable in small-wedge cases because of the limited coverage of reciprocal space, and final symmetry determination for merging is deferred until all wedges are processed.

When DIALS is selected, dials.index is executed several times until success is obtained by trying all indexing methods with and without the local indexing option. Finally, the integration result is converted to the XDS_ASCII.HKL format and saved as DIALS. HKL.

The processing status of individual wedges is displayed on the GUI (Fig. 2). In the top half, wedges are listed with rotation ranges, processing success or failure, estimated space group and resolution. The details of the processing results are shown below by clicking the list, including the statistics table, plots and $\log$ files. In small-wedge cases, the statistics for individual wedges are not very informative and users should proceed to merging without trying to select wedges based on the statistics.

\subsection{Preparation for merging}

2.3.1. Grouping results by lattice and deciding on the space group. Unit-cell parameters in $P 1$ symmetry (Niggli reduced cell) of all data sets are initially compared with each other by taking possible reindexing operators into account. An undirected graph is constructed where each node represents a data set and an edge is drawn if two unit-cell parameters are sufficiently similar. The connected components are extracted from the graph and sorted by population. Small connected components are usually ignored because in most cases such data sets are wrongly indexed. For each group, possible point-group symmetries are listed, which are calculated based on the averaged unit-cell parameter (Zwart et al., 2006). To help to decide the symmetry, the 'frequencies' (how often each symmetry was assigned by POINTLESS) are provided (Fig. 3). In our test cases, the most frequent symmetry was usually the correct one. Alternatively, users can

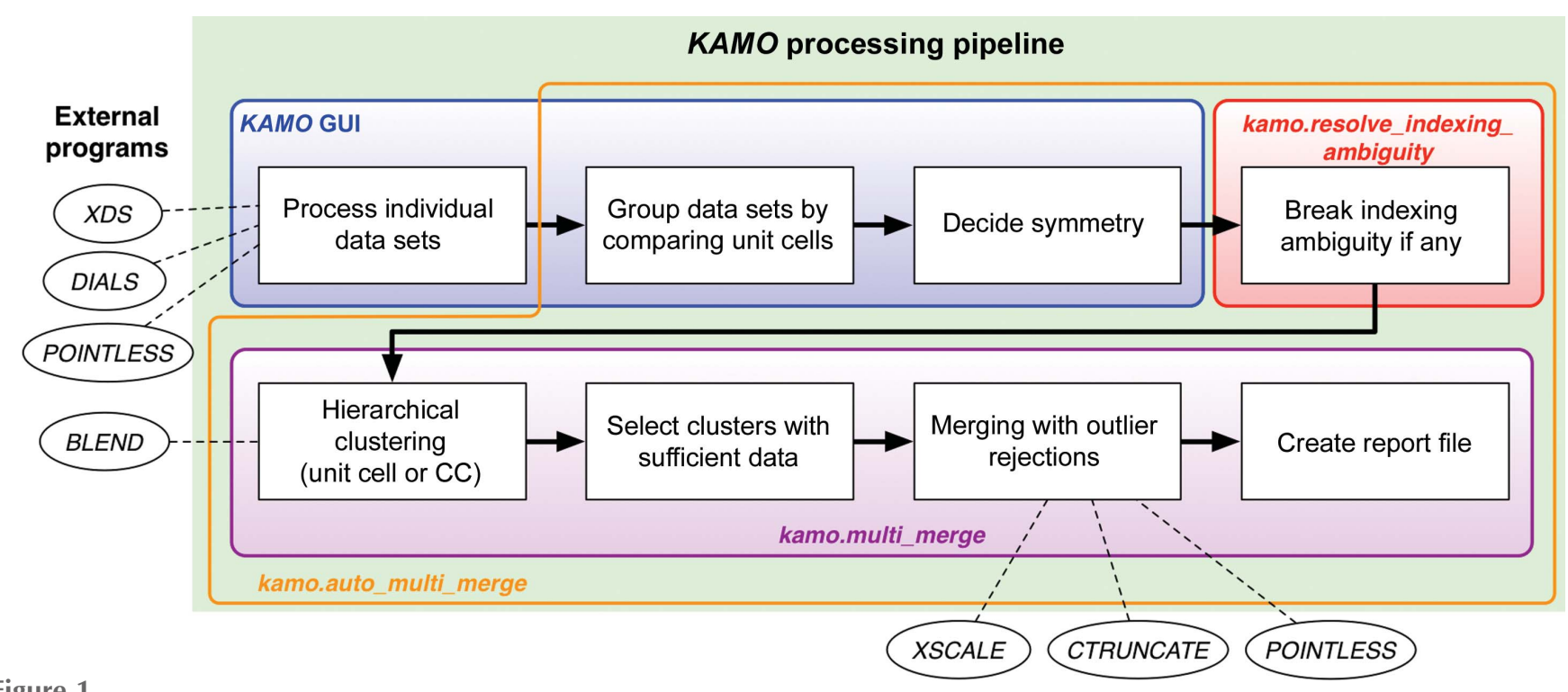

Figure 1

The workflow of $K A M O$ for multiple small-wedge data sets with the program name for each role provided. The external programs that are required are also shown. 


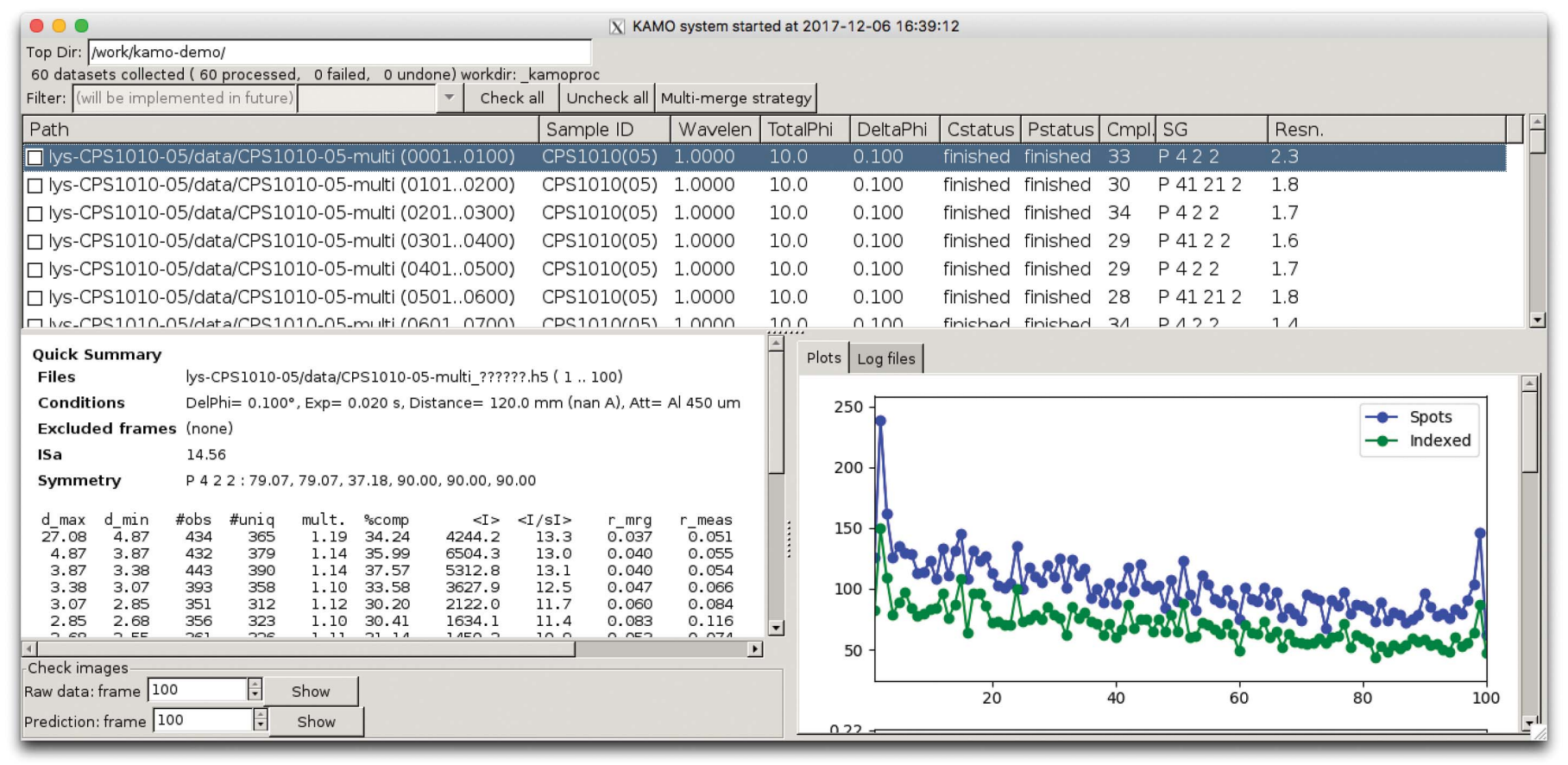

Figure 2

The KAMO GUI for processing individual wedges and for initiating the merging procedure. The collected data sets are listed with data-collection parameters and data-processing summaries. In the lower panel, the processing details for a selected item can be seen, including log files and plots with respect to image numbers.

assume a lower symmetry down to $P 1$, break indexing ambiguities and let POINTLESS decide the symmetry using the scaled and merged results. The automated symmetrydetermination procedure will be implemented in the future.

When the symmetry has been determined, the XDS ASCII.HKL_noscale files (see \$2.2) are reindexed to the specified unit-cell parameters and symmetry. The unit-cell parameters can optionally be refined by XDS (JOB= CORRECT) with constraints imposed by the selected Laue symmetry. New XDS_ASCII.HKL_noscale files are saved in the working directory for merging.

2.3.2. Breaking indexing ambiguity. There is ambiguity in the manner of indexing when the selected symmetry is lower than the highest possible symmetry. This always happens in point groups $23,6,32,3$ and 4 , but also happens in, for example, point groups 222 when $a \simeq b$ and 2 when $\beta \simeq 90^{\circ}$, as in (pseudo)merohedral twinning. The indexing modes need to be made consistent among all files by reindexing each data set appropriately. The simplest way to achieve this is to use isomorphous reference data, which have common reflections with all data sets. Without an external reference, one of the data sets may be used as a reference data set provided that it shares enough reflections with the others, but this may not be the case for small-wedge data because the number of reflections in each data set is limited. The situation is similar to serial crystallography, where this problem is well known as the 'indexing ambiguity' problem. There are a number of solutions, including the Brehm and Diederichs algorithm (Brehm \& Diederichs, 2014) and the selective breeding algorithm (Kabsch, 2014). The selective-breeding algorithm is implemented in yamtbx and is adopted as the default in kamo.resolve_indexing_ambiguity when an external isomorphous data set does not exist; otherwise the reference-based method can also be used in this command.

\section{4. kamo.multi_merge: clustering and merging}

After preparing data sets with similar unit-cell parameters by a consistent indexing mode, clustering and merging with outlier rejections can be initiated using kamo.multi_merge. There are two options in hierarchical clustering: the unit cellbased method using BLEND functions (Foadi et al., 2013) and the correlation coefficient (CC)-based method. The clusters are sorted by completeness and redundancy, and those that exceed a threshold ( $\geq 90 \%$ and $\geq 2$ by default, respectively) are subjected to merging and outlier-rejection cycles. The GUI prepares shell scripts for the two clustering methods, which

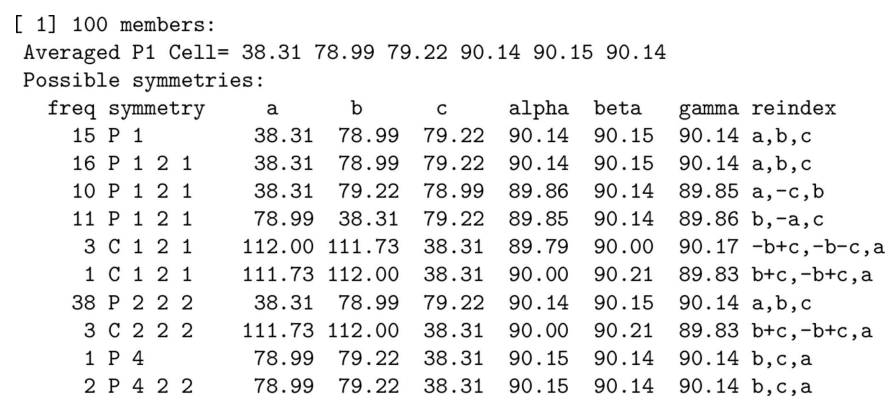

Figure 3

Grouping of indexed results based on unit-cell parameters in preparation for merging (titin data; simulated $1 \mathrm{~g} 1 \mathrm{c}$ ). In this case all 100 data sets were indexed with a consistent unit cell. The averaged cell in $P 1$ is shown and the possible point-group symmetries are listed with the 'frequency' of how many times POINTLESS assigned the symmetry. 
can easily be edited. Prior to the clustering analysis, data sets that have unit-cell parameters that are extremely different from the median values can be removed.

The CC-based clustering method uses a correlation coefficient of intensity that is calculated using the common reflections. $d(i, j)=[1-\mathrm{CC}(i, j)]^{1 / 2}$ is used as the distance between data sets $i$ and $j$ and hierarchical clustering analysis is performed with the Ward method (Murtagh \& Legendre, 2014). $\left[1-\mathrm{CC}^{2}(i, j)\right]^{1 / 2}$ can optionally be selected as used in Giordano et al. (2012). Data sets that have too few ( $<3$ by default) reflections in common with any other data set are removed before clustering. This threshold can be increased by specifying the Cc_clustering.min_common_refs option, which may stabilize $\mathrm{CC}$ calculation but reject more data sets. Data sets may have different intensity falloffs with respect to resolution, i.e. different overall $B$ values, which invalidates the calculation of $\mathrm{CC}$ over whole reflections. To mitigate this problem, there is an option to use intensities corrected using Wilson $B$ values or to use squared normalized structure-factor amplitudes $|E|^{2}$.

For each cluster, data sets belonging to a cluster are subjected to merging with outlier rejections. $X S C A L E$ is used for merging and outliers are detected by analyzing the XSCALE output. There are two kinds of outliers that should be removed, bad frames and bad crystals, both of which deteriorate the merging results. Bad frames may be identified when the crystal is out of the beam (often the first and last frames when incorrectly centred) or show decay owing to radiation damage, which is often the case in room-temperature experiments. Bad crystals (data sets) may be attributed to, for example, poorly performed integration with incorrect predictions and poor isomorphism. The merging and outlierrejection procedure is performed for up to three cycles. Initially, all data sets in the cluster are scaled and merged and bad frames are detected by comparing the intensities of each frame against the merged data. The bad frames are identified by a CC that is too small based on Tukey's $1.5 \times$ IQR criterion (Tukey, 1977) or a user-specified value. Secondly, bad data sets are detected from the merged result excluding bad frames. Several detection methods have been implemented using the error-model parameters $a b$ or $b$, the scaling parameter $B$ and the $R$ factor. The error-model parameters $a$ and $b$ are refined for each data set in XSCALE to correct the error of intensity $\sigma(I)$ through $\sigma^{2}(I)=a\left[\sigma_{\text {counting }}^{2}(I)+b I^{2}\right]$, and large $b$ or $a b$ values indicate that the data set has large systematic errors (Diederichs, 2010). By default, data sets with extreme values of $b$ or $B$ are removed. The remaining data sets are merged using the data set with the smallest intensity falloff with respect to resolution (the smallest $B$ in $X S C A L E$ ) as a scaling reference. However, in the first cycles a data set with median $B$ is used as a reference because using a data set with an extremely small $B$ as a reference sometimes results in useless statistics and merged data. This is probably because such data sets at extrema tend to be outliers and scaling against them can degrade scaling coefficients. The scaled intensity is converted to MTZ format using XDSCONV and analyzed using phenix.xtriage (Zwart et al., 2005) and CTRUNCATE (Winn et al., 2011). The MULTIPLICITY column is added to the MTZ file to check the multiplicity of each reflection. Diffraction anisotropy is analyzed with weighted $\mathrm{CC}_{1 / 2}$ along the principal axes using the same method as in AIMLESS (Evans \& Murshudov, 2013).

In XSCALE, three kinds of corrections are performed: decay, modulation and absorption. The $\mathrm{NBATCH}=$ parameter, which defines the number of scaling batches, is automatically determined by XSCALE by default; however, when severe radiation damage occurs it may help to increase $\mathrm{NBATCH}=$. In kamo.multi_merge, the number of frames per scaling batch can be controlled using either the xscale.frames_per_ batch or xscale.degrees_per_batch parameter, and a small number (for example one) should be given if radiation damage is severe.

The summary and details of clustering and merging results are reported in an HTML file with visualization using amCharts and D3.js (Supplementary Fig. S1).

\section{5. kamo.auto_multi_merge: fully automated merging for multiple samples}

kamo.multi_merge, as described above, is useful when processing data in a semi-automated manner by manually setting parameters to optimize the results. When users want to process many data sets of multiple samples at the same time in a fully automated manner, kamo.auto_multi_merge can be used. This program finds processed results from the KAMO GUI, deduces the space group, breaks the indexing ambiguity, runs kamo.multi_merge, determines the high-resolution cutoff for each sample and runs kamo.multi_merge once more with this cutoff. The high-resolution cutoff is determined by fitting the curve of $\mathrm{CC}_{1 / 2}$ versus resolution shells as used in AIMLESS and undergoes fine-tuning to ensure that the outershell $\mathrm{CC}_{1 / 2}$ is close to the specified value ( 0.5 by default). The result with the highest overall and outer-shell $\mathrm{CC}_{1 / 2}$ is considered to be the best result. Sample information can be prepared using a CSV file, where the root directory, sample names, anomalous flag and reference data file (optional) are specified. The report is an HTML file, which is generated to review all merging results at the same time.

\subsection{Availability}

The source code of $K A M O$ is available under the new BSD license at GitHub (https://github.com/keitaroyam/yamtbx). Documentation, including installation notes, is available at https:/github.com/keitaroyam/yamtbx/blob/master/doc/ kamo-en.md. Installation is easy when PHENIX or DIALS is already installed because they include most of the dependencies, including $c c t b x$ and wxPython.

\section{Examples}

$K A M O$ has been used for several structure analyses (Table 1). Processing notes describing the analysis of raw data that are publicly available can be found at https:/github.com/ keitaroyam/yamtbx/wiki. In this section, some processing 
Table 1

Current structure analyses that $K A M O$ has contributed to.

\begin{tabular}{|c|c|c|c|c|c|c|c|c|}
\hline Sample & $\begin{array}{l}\text { Polyhedra } \dagger \\
\text { (wild type) }\end{array}$ & $\begin{array}{l}\text { Polyhedra } \dagger \\
\text { (mutant) }\end{array}$ & $\mathrm{LPA}_{6} \ddagger$ & $\mathrm{ET}_{\mathrm{B}} \mathrm{R} \S$ & $\begin{array}{l}\text { TPT ब } \\
\left(\mathrm{P}_{\mathrm{i}}\right)\end{array}$ & $\begin{array}{l}\text { TPT } \\
\text { (3-PGA) }\end{array}$ & AtDTX14 $+\dagger$ & $\mathrm{OX} 2 \mathrm{R}+\neq$ \\
\hline PDB code & $5 \mathrm{gqm}$ & $5 \mathrm{gqn}$ & $5 \mathrm{xsz}$ & $5 \mathrm{xpr}$ & $5 \mathrm{y} 78$ & $5 \mathrm{y} 79$ & $5 \mathrm{y} 50$ & $5 \mathrm{wqc}$ \\
\hline Resolution $(\AA)$ & 1.68 & 1.55 & 3.2 & 3.5 & 2.1 & 2.2 & 2.6 & 1.96 \\
\hline Degrees per data set & 5 & 5 & 4,6 & 10 & $3-10,30$ & $3-10$ & $5,10,20$ & $1-6$ \\
\hline No. of data sets collected & 20 & 184 & 397 & 16 & 723 & 332 & 373 & 805 \\
\hline No. of data sets processed for merging & 20 & 155 & 350 & 16 & 599 & 250 & 139 & 768 \\
\hline
\end{tabular}

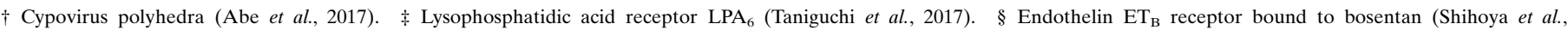

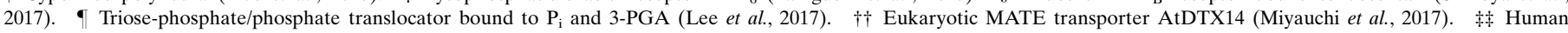
orexin 2 receptor (Suno et al., 2017).

examples are presented using the methods described here. The program versions used here were yamtbx commit 1107fc4 (as of 26 December 2017) with cctbx commit 16b2e7c (as of 17 April 2017); XDS 1 May 2016 (BUILT=20160617); $C C P 47.0$ (update 042); PHENIX 1.11.1; SHELXC, SHELXD and SHELXE 2016/1, 2013/2 and 2016/3, respectively; and $R$ 3.2.2. For data processing using $X D S$, diffraction images in the HDF5 format were read through the modified version of Takanori Nakane's eiger2cbf (https:/github.com/keitaroyam/ eiger2cbf). The processing results may differ slightly from the original studies because of the use of different versions.

All data sets except for the synthetic data were collected at BL32XU, SPring-8, Hyogo, Japan.

\subsection{James Holton's microfocus data-processing challenge}

James Holton opened 'The Micro-focus Data Processing Challenge' (http://bl831.als.lbl.gov/ jamesh/challenge/microfocus) using simulated diffraction images of titin (PDB entry $1 \mathrm{~g} 1 \mathrm{c})$ with an anomalous signal from selenomethionine at $0.9793 \AA$ wavelength. We downloaded the minimal version of the data, which consisted of 100 wedges of $3^{\circ}$. These synthetic data have two issues: severe radiation damage and indexing ambiguity caused by the space group, $P 2_{1} 2_{1} 2_{1}$ with $b \simeq c$ ( $a=38.3, b=78.6, c=79.6 \AA)$.

Here, we demonstrate automatic data processing, spacegroup determination, resolution of indexing ambiguity and merging using $K A M O$ followed by successful SAD phasing. The $K A M O$ GUI was started with default parameters and all 100 data sets were processed by $X D S$ without failure. The most frequent point-group symmetry determined by POINTLESS for each data set was P222 (38 times; Fig. 3). All integration results were reindexed to $P 222$ symmetry. The indexing ambiguity was resolved by kamo.resolve_indexing_ ambiguity with the selective breeding algorithm in two cycles, which was later validated using the $1 \mathrm{~g} 1 \mathrm{c}$ data (Fig. 4). kamo.multi_merge was then used for clustering and merging with outlier rejections at $1.8 \AA$ resolution (the detector edge). Clustering was found to be unnecessary because the largest cluster (using all data) resulted in the best statistics and phase quality. As radiation damage was severe, the option xscale.degrees_per_batch $=1$ to increase the number of scaling batches so that one batch corresponded to $1^{\circ}$ was essential for the success of SAD phasing, and also gave better data statistics and higher anomalous difference Fourier peak heights (Fig. 5). The data-processing, phasing and refinement statistics with a comparison with the non-KAMO results are summarized in Supplementary Table S1. Determination of the heavy-atom sites, phasing and phase improvement were performed with $S H E L X C / D / E$ (Sheldrick, 2010). All four heavy-atom sites were correctly located by SHELXD and 157 polyalanine residues were built by SHELXE in four cycles. The resulting CC against the native data was $39.45 \%$.

\section{2. $\mathrm{M}_{2}$ receptor: $\mathrm{Hg}-\mathrm{SAD}$ phasing}

Here, a real SAD phasing case is demonstrated using the human muscarinic acetylcholine receptor $M_{2}\left(M_{2} R\right) .671$ small-wedge $\left(5^{\circ}\right.$ per crystal) data sets were collected from mercury-bound $\mathrm{M}_{2} \mathrm{R}$-BRIL crystals (PDB entry 5yc8) using

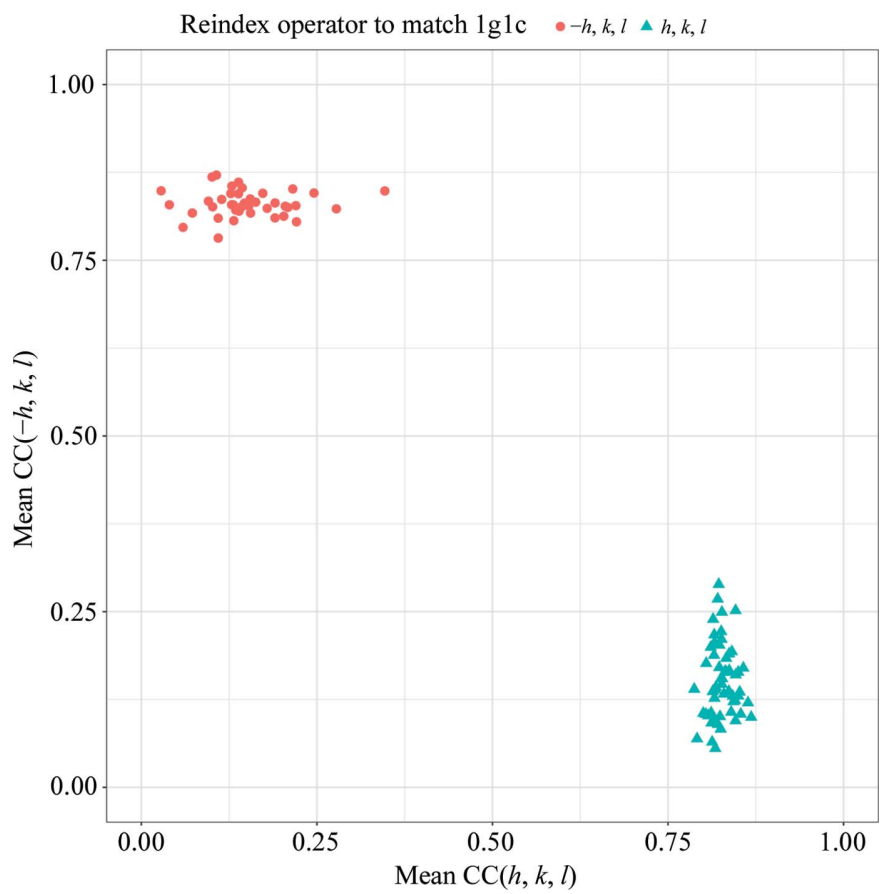

Figure 4

Breaking indexing ambiguity in the titin data (simulated $1 \mathrm{~g} 1 \mathrm{c}$ ). The averaged $\mathrm{CC}$ with all other wedges in the final (converged) cycle is plotted for two possible modes $(h, k, l$ and $-h, l, k)$. The resolution was validated using the original $1 \mathrm{~g} 1 \mathrm{c}$ data (shown as symbols and colours). This figure was prepared using ggplot2 (Wickham, 2009) in $R$ ( $\mathrm{R}$ Development Core Team, 2008). 
the $Z O O$ system and an MX225-HS CCD detector (Rayonix) to obtain model bias-free phase information by SAD phasing (Suno et al., manuscript in preparation). Exposure conditions were automatically adjusted so that each crystal absorbed 7-12 MGy. The expected anomalous signal $\left\langle\left|\Delta F_{\text {ano }}\right|\right\rangle /\langle|F|\rangle$ was $4.2 \%$ at $1 \AA$ wavelength with full occupancies, which was

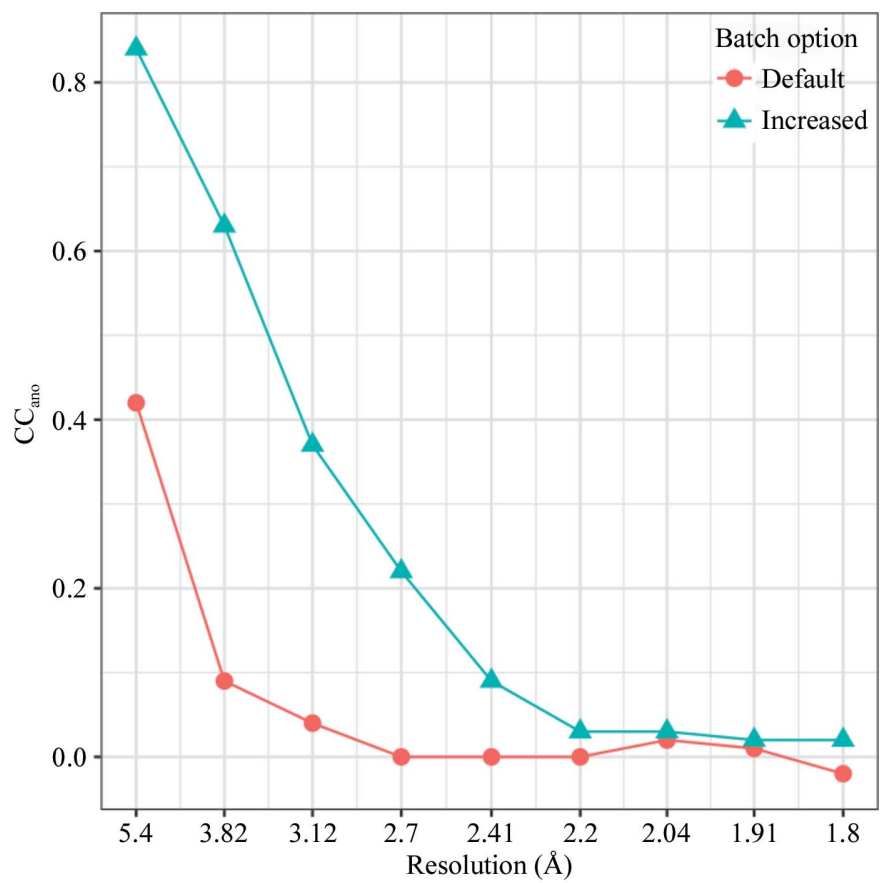

(a)

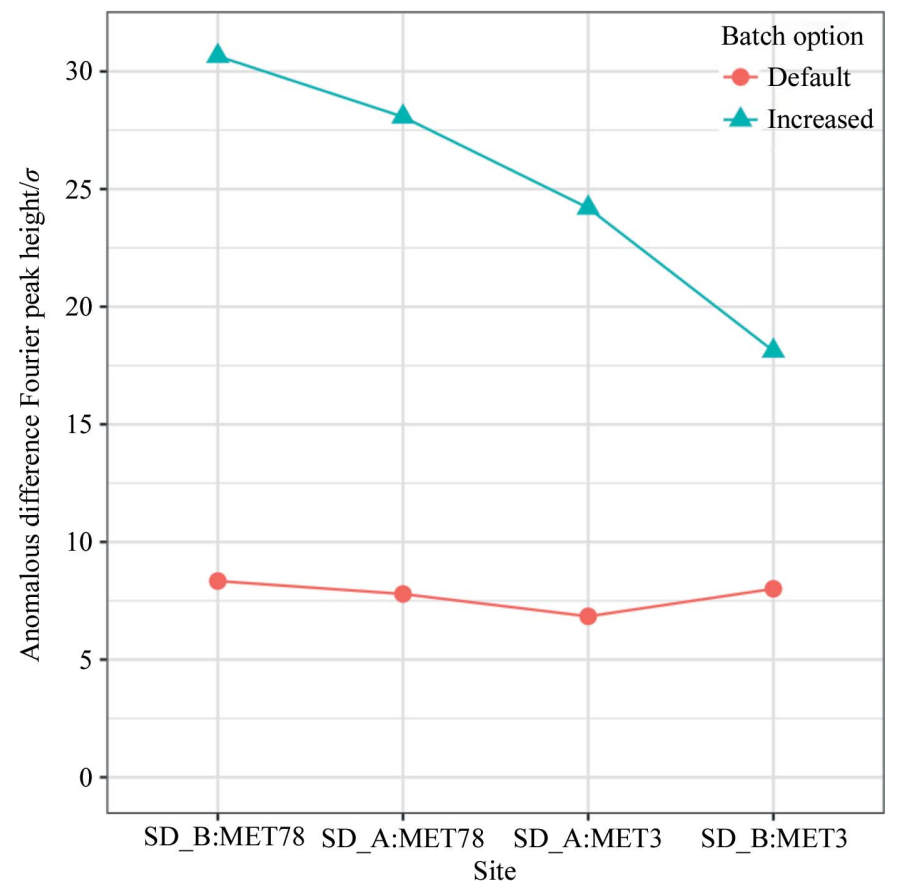

Figure 5

(b)

Improved anomalous data quality by increasing the scaling batches in the titin data (simulated $1 \mathrm{~g} 1 \mathrm{c}$ ). To increase the number of batches the option xscale.degrees_per_batch=1 was given in kamo.multi_merge. (a) $\mathrm{CC}_{\mathrm{ano}}$ is the correlation coefficient of $I^{(+)}-I^{(-)}$between random halfsets. (b) Anomalous difference Fourier peak heights calculated using the observed anomalous differences and the $1 \mathrm{~g} 1 \mathrm{c}$ model with SHELXC and ANODE (Thorn \& Sheldrick, 2011). calculated using the equation of Hendrickson \& Teeter (1981). The actual anomalous signal was estimated to be $3.2 \%$ based on the $F_{\text {model }}$ of the refined structure. The raw diffraction images will be made available in the Zenodo data repository (https://doi.org/10.5281/zenodo.1172266) when the associated manuscript is published.

The $K A M O$ GUI was started with default parameters, and 547 data sets were indexed and integrated using XDS. Of these, 532 data sets had consistent unit-cell parameters, with the most frequent (341 times) point-group symmetry being $P 2$; the data sets were thus reindexed in $P 2$. Clustering by unit-cell parameters was performed using $B L E N D$. SAD phasing was performed with the $S H E L X C / D / E$ programs (Sheldrick, 2010). 10000 trials to find three mercury sites were performed using SHELXD and 30 cycles of polyalanine tracing after phase improvement assuming $55 \%$ solvent content were performed with SHELXE. From the largest cluster consisting of all data sets, 238 polyalanine residues were built with a CC of $39.03 \%$ by SHELXE in 24 cycles and the electron-density map was sufficiently clear to trace the $\mathrm{M}_{2} \mathrm{R}$ structure. The data processing, phasing and refinement statistics with a comparison with the non-KAMO results are summarized in Supplementary Table S2.

To compare clusters, the real-space CC of the SAD-phased electron-density map against the map calculated from the $5 \mathrm{yc} 8$ model $\left(\mathrm{CC}_{\text {map }}\right)$ was calculated for each cluster using phenix.get_cc_mtz_pdb (Adams et al., 2010). The correctness of the located $\mathrm{Hg}$ sites and $\mathrm{CC}_{\text {map }}$ are summarized in Table 2 along with representative statistics and unit-cell variations. The largest cluster had the best phase quality despite large unit-cell variations. CC-based clustering was also attempted; however, no subcluster offering better quality was found.

\subsection{Cypovirus polyhedra: clustering}

Here, the power of clustering to achieve a better result is demonstrated using cypovirus polyhedra, a natural crystalline protein assembly of the polyhedrin monomer produced in infected insect cells (Abe et al., 2017). 184 small-wedge $\left(5^{\circ}\right)$ data sets for a deletion mutant $\triangle 3$ (PDB entry $5 \mathrm{gqn}$ ) were collected automatically using $Z O O$ and an EIGER X $9 \mathrm{M}$ detector (DECTRIS). Exposure conditions were automatically adjusted so that each crystal absorbed 11.6 MGy. The crystals belonged to space group I23, with unit-cell parameter $a \simeq 103 \AA$. The raw diffraction images are available from the Zenodo data repository (https://doi.org/10.5281/ zenodo.846473).

The $K A M O$ GUI was started with default parameters and 157 data sets were successfully indexed and integrated with a consistent unit cell. The most frequent (122 times) symmetry was $I 23$, which was consistent with the known structure (PDB entry 2oh6; Coulibaly et al., 2007). The indexing ambiguity was resolved using the known isomorphous reference data (PDB entry 2oh6) by kamo.resolve_indexing_ambiguity.

For merging, kamo.multi_merge was used to $1.55 \AA$ resolution and several clustering metrics, $B L E N D, \mathrm{CC}(I)$ and $\mathrm{CC}\left(|E|^{2}\right)$, were compared. Structure refinement was 
Table 2

Data and SAD phase quality for clusters by unit-cell similarities using BLEND for mercury-bound $\mathrm{M}_{2} \mathrm{R}$ data (PDB entry 5yc8).

The top eight results sorted by multiplicity are shown. LCV is the linear cell variation defined in BLEND (Foadi et al., 2013). The inner and outer resolution ranges are 50-7.50 and 2.65-2.50 А, respectively. The correctness of the located $\mathrm{Hg}$ sites was evaluated against those of the 5yc8 model using phenix.emma (Adams et al., 2002). $B_{\text {Wilson }}$ is the Wilson $B$ value reported by CTRUNCATE. Completeness is greater than or equal to $98 \%$ for every case. For the calculation of multiplicity, $\mathrm{CC}_{1 / 2}$ and $\mathrm{CC}_{\mathrm{ano}}$, Friedel pairs are treated as different reflections.

\begin{tabular}{|c|c|c|c|c|c|c|c|c|c|}
\hline \multirow[b]{2}{*}{ LCV (\%) } & \multirow[b]{2}{*}{ Cluster height } & \multirow[b]{2}{*}{ No. of data sets merged } & \multirow[b]{2}{*}{ Multiplicity } & \multirow[b]{2}{*}{$\mathrm{CC}_{1 / 2}$ (outer) } & \multirow[b]{2}{*}{$\mathrm{CC}_{\text {ano }}$ (inner) } & \multicolumn{2}{|c|}{$\mathrm{Hg}$ correctly located } & \multirow[b]{2}{*}{$\mathrm{CC}_{\text {map }}$} & \multirow[b]{2}{*}{$B_{\text {Wilson }}\left(\AA^{2}\right)$} \\
\hline & & & & & & No. & R.m.s.d. (Å) & & \\
\hline 19.6 & 88.56 & 454 & 20.7 & 0.611 & 0.80 & 3 & 0.60 & 0.575 & 33.8 \\
\hline 4.6 & 56.42 & 388 & 17.7 & 0.582 & 0.76 & 3 & 1.16 & 0.506 & 33.3 \\
\hline 4.6 & 48.77 & 283 & 13.0 & 0.460 & 0.67 & 3 & 1.27 & 0.505 & 34.1 \\
\hline 4.0 & 42.69 & 215 & 10.0 & 0.379 & 0.63 & 3 & 0.75 & 0.415 & 33.7 \\
\hline 4.0 & 28.15 & 184 & 8.6 & 0.349 & 0.59 & 2 & 0.67 & 0.317 & 33.7 \\
\hline
\end{tabular}

Table 3

Comparison of clustering methods using polyhedra data (PDB entry 5gqn).

The top 12 results sorted by $R_{\text {free }}$ are shown out of 99 clusters tested. The inner and outer resolution ranges are $50-4.65$ and $1.65-1.55 \AA$, respectively. $\langle B\rangle$ is the averaged atomic $B$ value reported by phenix.refine. Completeness is greater than or equal to $99.8 \%$ for every case.

\begin{tabular}{|c|c|c|c|c|c|c|c|c|}
\hline Clustering method & $\operatorname{LCV}(\%)$ & No. of data sets merged & Multiplicity & $\mathrm{CC}_{1 / 2}$ (inner) & $\mathrm{CC}_{1 / 2}$ (outer) & $R_{\text {work }}$ & $R_{\text {free }}$ & $\langle B\rangle\left(\AA^{2}\right)$ \\
\hline $\mathrm{CC}\left(|E|^{2}\right)$ & 0.4 & 18 & 9.7 & 0.989 & 0.753 & 0.1411 & 0.1765 & 10.7 \\
\hline $\mathrm{CC}\left(|E|^{2}\right)$ & 0.4 & 15 & 7.7 & 0.985 & 0.734 & 0.1436 & 0.1797 & 9.7 \\
\hline $\mathrm{CC}\left(|E|^{2}\right)$ & 0.9 & 24 & 13.1 & 0.941 & 0.805 & 0.1443 & 0.1807 & 10.5 \\
\hline $\mathrm{CC}(I)$ & 0.9 & 38 & 19.9 & 0.989 & 0.670 & 0.1447 & 0.1823 & 9.7 \\
\hline $\mathrm{CC}(I)$ & 0.4 & 22 & 11.9 & 0.983 & 0.627 & 0.1437 & 0.1825 & 10.6 \\
\hline $\mathrm{CC}(I)$ & 0.4 & 17 & 8.7 & 0.980 & 0.620 & 0.1459 & 0.1828 & 10.4 \\
\hline BLEND & 1.2 & 110 & 57.3 & 0.984 & 0.722 & 0.1481 & 0.1871 & 8.0 \\
\hline All data & 2.2 & 130 & 67.8 & 0.969 & 0.712 & 0.1551 & 0.1939 & 7.1 \\
\hline
\end{tabular}

performed for all merged results using phenix.refine starting from the 5gqn model with strategies of rigid-body, individual ADP and coordinate, and occupancy refinement. The top 12 results were sorted according to $R_{\text {free }}$ and are listed in Table 3. $\mathrm{CC}\left(|E|^{2}\right.$ ) gave the best cluster (multiplicity of 9.7) in $\mathrm{CC}_{1 / 2}$ and $R_{\text {free }}$ rather than merging all data sets even after outlier rejections (multiplicity of 67.8). The data-processing and refinement statistics with a comparison with the non-KAMO results are summarized in Supplementary Table S3.

The reason why clustering was not useful in the other data sets mentioned above may be partly attributable to the limited number of common reflections for the calculation of $\mathrm{CC}$ owing to lower space-group symmetries. Moreover, the accuracy of subclusters may be compromised because of insufficient multiplicity when the total amount of data is limited. However, if almost all crystals are isomorphous, merging more data sets would simply improve the data quality and resolution.

\section{Conclusions and outlook}

$K A M O$, an automated data-processing system for multiple microcrystals, has been developed and its use is spreading in facilities across the world. $K A M O$ dramatically simplifies the processing of multiple small-wedge data sets, working with $X D S$ and $C C P 4$ programs. Data collection from a number of crystals was highly beneficial for successful SAD phasing (\$3.2) and finding the high-quality subset of the whole data (§3.3), which were easily achieved through KAMO.

This system has been routinely used on BL32XU and often combined with the automated data-collection system $Z O O$, which enables a quick evaluation of the collected data sets. These systems enable high-throughput and high-resolution structure analyses of challenging targets, crystals of which are often difficult to grow to large sizes (Abe et al., 2017; Shihoya et al., 2017; Taniguchi et al., 2017; Lee et al., 2017; Miyauchi et al., 2017; Suno et al., 2017). In many cases, the default parameters of $K A M O$ are adjusted to result in sufficient data quality, but some fine-tuning may be required to obtain better quality, such as sampling several clustering methods. Future directions include better algorithms for clustering and outlier rejections, full automation without any human intervention, implementation of all functions in the GUI and on-the-fly merging functions. On-the-fly merging will provide the resolution and data quality of the collected data sets, and improve efficiency by aiding decision making on whether to stop the experiment and switch to another sample. 


\section{Acknowledgements}

The authors are grateful to R. Suno, T. Kobayashi and S. Iwata for making the $\mathrm{M}_{2}$ receptor data available to us. C. Gopalasingam, K. Hasegawa, G. Ueno, H. Ago, T. Nakane and H. E. Kato are acknowledged for their careful reading of the manuscript and valuable comments. KY thanks the Special Postdoctoral Researcher Program of RIKEN. We thank the Edanz Group (http://www.edanzediting.com/ac) for editing a draft of this manuscript.

\section{Funding information}

This work was supported by JSPS KAKENHI Grant No. 15H06862 and the Platform Project for Support in Drug Discovery and Life Science Research (Platform for Drug Discovery, Informatics and Structural Life Science) from the Japan Agency for Medical Research and Development (AMED).

\section{References}

Abe, S., Tabe, H., Ijiri, H., Yamashita, K., Hirata, K., Atsumi, K., Shimoi, T., Akai, M., Mori, H., Kitagawa, S. \& Ueno, T. (2017). ACS Nano, 11, 2410-2419.

Adams, P. D. et al. (2010). Acta Cryst. D66, 213-221.

Adams, P. D., Grosse-Kunstleve, R. W., Hung, L.-W., Ioerger, T. R., McCoy, A. J., Moriarty, N. W., Read, R. J., Sacchettini, J. C., Sauter, N. K. \& Terwilliger, T. C. (2002). Acta Cryst. D58, 1948-1954.

Akey, D. L., Brown, W. C., Konwerski, J. R., Ogata, C. M. \& Smith, J. L. (2014). Acta Cryst. D70, 2719-2729.

Assmann, G., Brehm, W. \& Diederichs, K. (2016). J. Appl. Cryst. 49, 1021-1028.

Brehm, W. \& Diederichs, K. (2014). Acta Cryst. D70, 101-109.

Caffrey, M. (2015). Acta Cryst. F71, 3-18.

Coulibaly, F., Chiu, E., Ikeda, K., Gutmann, S., Haebel, P. W., SchulzeBriese, C., Mori, H. \& Metcalf, P. (2007). Nature (London), 446, 97101.

Cusack, S., Belrhali, H., Bram, A., Burghammer, M., Perrakis, A. \& Riekel, C. (1998). Nature Struct. Biol. 5, 634-637.

Diederichs, K. (2010). Acta Cryst. D66, 733-740.

Diederichs, K. (2017). Acta Cryst. D73, 286-293.

Evans, P. R. (2011). Acta Cryst. D67, 282-292.

Evans, G., Axford, D., Waterman, D. \& Owen, R. L. (2011). Crystallogr. Rev. 17, 105-142.

Evans, P. R. \& Murshudov, G. N. (2013). Acta Cryst. D69, 1204-1214.

Foadi, J., Aller, P., Alguel, Y., Cameron, A., Axford, D., Owen, R. L., Armour, W., Waterman, D. G., Iwata, S. \& Evans, G. (2013). Acta Cryst. D69, 1617-1632.

Fraser, J. S., van den Bedem, H., Samelson, A. J., Lang, P. T., Holton, J. M., Echols, N. \& Alber, T. (2011). Proc. Natl Acad. Sci. USA, 108, $16247-16252$.

Giordano, R., Leal, R. M. F., Bourenkov, G. P., McSweeney, S. \& Popov, A. N. (2012). Acta Cryst. D68, 649-658.

Grosse-Kunstleve, R. W., Sauter, N. K., Moriarty, N. W. \& Adams, P. D. (2002). J. Appl. Cryst. 35, 126-136.

Hagberg, A. A., Schult, D. A. \& Swart, P. J. (2008). Proceedings of the 7th Python in Science Conference (SciPy 2008), edited by G. Varoquaux, T. Vaught \& J. Millman, pp. 11-15. http:// conference.scipy.org/proceedings/scipy2008/paper_2/.

Hendrickson, W. A. \& Teeter, M. M. (1981). Nature (London), 290, 107-113.

Huang, C.-Y., Olieric, V., Ma, P., Howe, N., Vogeley, L., Liu, X., Warshamanage, R., Weinert, T., Panepucci, E., Kobilka, B.,
Diederichs, K., Wang, M. \& Caffrey, M. (2016). Acta Cryst. D72, 93-112.

Huang, C.-Y., Olieric, V., Ma, P., Panepucci, E., Diederichs, K., Wang, M. \& Caffrey, M. (2015). Acta Cryst. D71, 1238-1256.

Jones, E. et al. (2001). SciPy: Open Source Scientific Tools for Python. http://www.scipy.org.

Kabsch, W. (2010). Acta Cryst. D66, 125-132.

Kabsch, W. (2014). Acta Cryst. D70, 2204-2216.

Lee, Y., Nishizawa, T., Takemoto, M., Kumazaki, K., Yamashita, K., Hirata, K., Minoda, A., Nagatoishi, S., Tsumoto, K., Ishitani, R. \& Nureki, O. (2017). Nature Plants, 3, 825-832.

Liu, Q. \& Hendrickson, W. A. (2015). Curr. Opin. Struct. Biol. 34, 99 107.

Miyauchi, H., Moriyama, S., Kusakizako, T., Kumazaki, K., Nakane, T., Yamashita, K., Hirata, K., Dohmae, N., Nishizawa, T., Ito, K., Miyaji, T., Moriyama, Y., Ishitani, R. \& Nureki, O. (2017). Nature Commun. 8, 1633.

Murtagh, F. \& Legendre, P. (2014). J. Classif. 31, 274-295.

Olieric, V., Weinert, T., Finke, A. D., Anders, C., Li, D., Olieric, N., Borca, C. N., Steinmetz, M. O., Caffrey, M., Jinek, M. \& Wang, M. (2016). Acta Cryst. D72, 421-429.

Owen, R. L., Juanhuix, J. \& Fuchs, M. (2016). Arch. Biochem. Biophys. 602, 21-31.

Parkhurst, J. M., Brewster, A. S., Fuentes-Montero, L., Waterman, D. G., Hattne, J., Ashton, A. W., Echols, N., Evans, G., Sauter, N. K. \& Winter, G. (2014). J. Appl. Cryst. 47, 1459-1465.

$\mathrm{R}$ Development Core Team (2008). R: A Language and Environment for Statistical Computing. Vienna: R Foundation for Statistical Computing.

Schlichting, I. (2015). IUCrJ, 2, 246-255.

Sheldrick, G. M. (2010). Acta Cryst. D66, 479-485.

Shihoya, W., Nishizawa, T., Yamashita, K., Inoue, A., Hirata, K., Kadji, F. M. N., Okuta, A., Tani, K., Aoki, J., Fujiyoshi, Y., Doi, T. \& Nureki, O. (2017). Nature Struct. Mol. Biol. 24, 758-764.

Smith, J. L., Fischetti, R. F. \& Yamamoto, M. (2012). Curr. Opin. Struct. Biol. 22, 602-612.

Suno, R. et al. (2017). Structure, 26, 7-19.

Taniguchi, R. et al. (2017). Nature (London), 548, 356-360.

Thorn, A. \& Sheldrick, G. M. (2011). J. Appl. Cryst. 44, 12851287.

Tukey, J. W. (1977). Exploratory Data Analysis. Reading: AddisonWesley.

Ueno, G., Kanda, H., Kumasaka, T. \& Yamamoto, M. (2005). J. Synchrotron Rad. 12, 380-384.

Vonrhein, C., Flensburg, C., Keller, P., Sharff, A., Smart, O., Paciorek, W., Womack, T. \& Bricogne, G. (2011). Acta Cryst. D67, 293302.

Wickham, H. (2009). ggplot2: Elegant Graphics for Data Analysis. New York: Springer.

Winn, M. D. et al. (2011). Acta Cryst. D67, 235-242.

Winter, G. (2010). J. Appl. Cryst. 43, 186-190.

Winter, G., Waterman, D. G., Parkhurst, J. M., Brewster, A. S., Gildea, R. J., Gerstel, M., Fuentes-Montero, L., Vollmar, M., MichelsClark, T., Young, I. D., Sauter, N. K. \& Evans, G. (2018). Acta Cryst. D74, 85-97.

Yamamoto, M., Hirata, K., Yamashita, K., Hasegawa, K., Ueno, G., Ago, H. \& Kumasaka, T. (2017). IUCrJ, 4, 529-539.

Zander, U., Bourenkov, G., Popov, A. N., de Sanctis, D., Svensson, O., McCarthy, A. A., Round, E., Gordeliy, V., Mueller-Dieckmann, C. \& Leonard, G. A. (2015). Acta Cryst. D71, 2328-2343.

Zander, U., Cianci, M., Foos, N., Silva, C. S., Mazzei, L., Zubieta, C., de Maria, A. \& Nanao, M. H. (2016). Acta Cryst. D72, 1026-1035.

Zwart, P. H., Grosse-Kunstleve, R. W. \& Adams, P. D. (2005). CCP4 Newsl. Protein Crystallogr. 43, contribution 7.

Zwart, P. H., Grosse-Kunstleve, R. W. \& Adams, P. D. (2006). CCP4 Newsl. Protein Crystallogr. 44, contribution 7. 